\title{
The Research of Group Mobility Model Based on Spectral Clustering Algorithm in Opportunistic Networks
}

\author{
He Hong \\ College of computer and communication, Hunan institute of engineering, Xiangtan, 411104,China \\ Email:744469708@qq.com \\ Wang Ning \\ College of computer and communication, Hunan institute of engineering, Xiangtan, 411104,China \\ Email:14880047@qq.com
}

\begin{abstract}
The research on group mobility in the opportunity network concerns what nodes' mobility may bring about, in contrast to the preciously-proposed group mobility approaches that are unilateral in the thoughts of the constitution of groups by nodes, resulting in, the distortion of reality to reflect group behavior characteristics that are revealed in the situations of their application. Based on the Spectral Clustering algorithm, this paper proposes the group mobility model (SCM), which takes the advantage of node's own properties, such as its spatial position, to set up node's affinity matrix, extract main characteristics from arbitrarily distributed nodes with the aid of spectral clustering algorithm, and regarding the needs of a task assigned cluster nodes dynamically in a fixed time. The model depicts truly and effectively the group characteristics of node's mobility, accomplish the conducts of group mobility and clustering well.
\end{abstract}

Key words - Opportunistic network, Spectral Clustering, group mobility model

\section{INTRODUCTION}

\begin{abstract}
Along with the thoroughly application of wireless network and the expansion of the scale day by day, the wireless network is often split and disconnected in some networks due to the rapid mobility of nodes or the destruction of nodes. When problems such as wireless network fragmentation and frequent connection interruption occur in such special environment, the traditional ad hoc wireless network[1][2] cannot operate effectively, network performance can degrade significantly and even crash.
\end{abstract}

Opportunity Network[3][4]has the common characteristics of Delay Tolerant Network (DTN). It is a new type of self-organizing network that uses the encounter of nodes during the move to support data forwarding. Its transmission has several remarkable characteristics [5]; there is no end-to-end stable link between the sending end and the receiving end, and the network topology changes greatly; the data forwarding in the network is established by the accidental meeting of nodes and the delay is big. It is a special network with heterogeneous characteristics, which means all kinds of data transmission devices such as mobile phone, PDA or mobile base station can be regarded as mobile nodes.

Over the past decade, researchers have tried to observe and explore opportunity networks from various fields [6]. This paper focuses on what kind of influence the group mobility model in opportunity networks has on its performance. The group mobility model of opportunity network reflects the mobility characteristics and behavior characteristics of the group node. The state and mobility mode of the mobile node are constrained by other nodes. For example, the exponential correlation random mobility model [7]realizes different group mobility through the control of function parameters. The group mobility model of reference points 
[8]sets the reference center point, and the mobility mode of the whole group changes according to the mobility of the logical center point. Group force mobility model[9], combined with the conceptual model of social force, proposed three types of "virtual" group forces that obey the exponential distribution to adjust the mobility of all group nodes. In the group mobility model of reference region [10], each group is associated with the reference region, and the nodes in the group move towards the region. When all the nodes arrive, a new reference region is generated. Event-driven group mobility model [11] is a mobility model based on node trajectories, which mainly describes large-scale node mobility and is driven by events. At present, most group mobility models focus on the description of mobility characteristics. Considering that the network nodes constitute a single group, they cannot reflect the group behavior characteristics of the real map, or they cannot gather dynamically at a specific time or place, and the group behavior characteristics in motion are ignored, so they cannot operate properly.

In order to truly express the group mobility characteristics of the node, and effectively improve network performance, this paper proposes an opportunity network group mobility model (SCM) based on spectral clustering algorithm. By extracting the location information of nodes and analyzing it and making clustering division, the nodes on the map based on the real geographical environment can perform group concentration behavior and group mobility at specific time according to the needs.

\section{GROUP MOBILITY MODEL OF SPECTRAL CLUSTERING ALGORITHM}

The early clustering algorithms simply consider the similarity of two samples by constructing the similarity matrix of different feature structures. The algorithm has limitations in many aspects, and the clustering effect is not good. For example, the node distribution must be subject to the k-means algorithm of Gaussian distribution. Spectral clustering algorithm can improve these shortcomings, and clustering can be done for any shape of distribution. The spectral clustering algorithm obtains the corresponding similarity matrix by calculating the Gaussian kernel function, in which each vertex represents the similarity between the corresponding nodes. The whole similar matrix can also be regarded as an undirected graph, and then data mining can be realized by processing the graph.

\subsection{NORMALIZED SPECTRAL CLUSTERING ALGORITHM}

\section{BASED ON $L_{s y m}$ (NJW ALGORITHM)}

Jordan et al. [12] first proposed to preprocess the original data and construct the normalized Laplacian matrix $L_{s y m}$ according to the theory of spectral clustering algorithm. The feature vectors are extractedcorresponding to the first $\mathrm{k}$ minimum eigenvalues, and a $n \times k$ vector matrix space is established while still maintaining the corresponding relationship with the original sample.As a new relational matrix between node objects, each row of the matrix represents an object sample. This process describes the "dimensionality reduction" of the sample space data and the extraction of its main features. Finally, traditional classical clustering algorithms, such as k-means, CLARA and CHAMELEON, are used for clustering to obtain $\mathrm{k}$ categories of the final result. This algorithm is very flexible so other classical algorithms can still play their strengths when applied to do underlying clustering. Compared with the classical clustering algorithm, NJW algorithm[13]has a greater capacity for dealing with non-convex spherical data and higher clustering accuracy.

\subsection{GROUP MOBILITY METHOD BASED ON SPECTRAL CLUSTERING ALGORITHM}

The group mobility model of opportunity network studied in this paper can be modeled in combination with the basic theory of spectral clustering algorithm. The mobile node in the opportunity network itself can be regarded as the person who carries the intelligent mobile device. The spectrum clustering algorithm is used to cluster the group of people in the real environment and the behavior of group mobility. This is exactly realization and expansion of the application of the opportunity network under the specific environment requirements, so it needs to be constructed and analyzed.

The basic theory of spectral clustering algorithm is used to describe the behavior characteristics of network groups. According to the characteristics of different requirements and scenarios, the mobile behavior of the mobile model can be divided into the following situations: 
(1)Dispersed mobility behavior of entity nodes: The node mobility mode refers to the entity mobility model (SPMB) [14], which shows the process of random mobility on the map.

(2) Node aggregation group mobility behavior: The node mobility mode refers to the SCM mobility model proposed in this paper, calculates and goes to the corresponding clustering central point for aggregation, and then follows the central point for group mobility. The group central node still moves according to the original individual mobility model.

In this paper, $x_{1}, x_{2}, \cdots, x_{n}$ represent $\mathrm{n}$ nodes in the $\mathrm{ONE}$ opportunity network simulator. Each node is given a number, such as node 1 , node $2, \ldots$, node $n$. Each node is an intelligent mobile device carried by people, with a short distance communication range. Besides, the situation and coordinates of other nodes in the adjacent region can be known by probing, and the nodes communicate through the network interface.

Here, the process of applying the spectral clustering algorithm to the group mobility model is as follows:

(1) Set the number of clusters $\mathrm{k}$, the starting time of clustering $\mathrm{t}$, and generate $\mathrm{n}$ moving nodes $S=\left\{s_{1}, s_{2}, \cdots, s_{n}\right\}$. At the beginning of the simulation, n nodes move randomly on the map and do not affect each other until the specified clustering time $t$.

(2) Formula (1) is used to calculate the similarity matrix up to the specified starting time of aggregation $t$

$$
A(i, j)=\exp \left(-\frac{d^{2}\left(s_{i}, s_{j}\right)}{2 \sigma^{2}}\right)
$$

Where $i=j, A(i, i)=0$, and $\sigma$ is the scale parameter, which is set manually in the traditional algorithm. $d\left(s_{i}, s_{j}\right)$ represents the spatial distance of $s_{i}$ and $s_{j}$. Here, the two-dimensional Euclidean distance (formula 2) is used to solve it.

$$
d\left(x_{i 1}-x_{j 1}\right)=\sqrt{\left(x_{i 1}-x_{j 1}\right)^{2}+\left(x_{i 2}-x_{j 2}\right)^{2}}
$$

Since the selection of $\sigma$ has an impact on the clustering effect and it is difficult for manual selection to achieve a satisfactory effect, an adaptive parameter selection method is adopted according to the idea of self-tuning spectrum clustering [15] proposed by Perona et al. The algorithm proposes the idea of "Local Scaling". Different parameters are selected for each node locally, and each node $s_{i}$ corresponds to one $\sigma_{i}$. Therefore, the similarity matrix is calculated by the following formula (3):

$$
A(i, j)=\exp \left(-\frac{d^{2}\left(s_{i}, s_{j}\right)}{2 \sigma_{i} \sigma_{j}}\right)
$$

Where $\sigma_{i}=d\left(s_{i}, s_{W}\right)$ and $s_{W}$ represents the W-th nearest neighbor of the node $S_{i}$. By experience, generally when $W=7$ the algorithm works best, especially in the high-dimensional data.

(3) Construct the matrix $D$ as the degree matrix, where the elements $D(i, i)$ in the main diagonal position are the sum of the elements in the $\mathrm{i}$-th row of the similar matrix $A$ , and the values in other positions are all 0 . Then solvefor the Laplace matrix $L_{s y m}=D^{-1 / 2} L D^{-1 / 2}=I-D^{-1 / 2} A D^{-1 / 2}$.

(4)The feature vectors corresponding to $\mathrm{k}$ minimum eigenvalues of the matrix $L_{s y m}$ are solved as $x_{1}, x_{2} \cdots, x_{n}$ , and then the matrix $X=\left[x_{1}, x_{2}, \cdots, x_{k}\right] \in R^{n * k}$ is constructed, where the feature vectors are stored by column.

(5) Normalize the row vectors of the matrix $X$ to get the matrix $Y$, where:

$$
Y_{i j}=\frac{X_{i j}}{\left(\sum_{j} X_{i j}^{2}\right)^{1 / 2}}
$$

(6) "Dimensionality reduction" has been completed here. Each row of the matrix $Y$ is regarded as a node in space $R^{k}$, any traditional clustering algorithm can be used for clustering calculation (k-means). 
(7) If the data of the i-th row of the matrix $Y$ falls into the $\mathrm{j}$-th category, it means that the original data node $S_{i}$ is also divided into the $\mathrm{j}$-th category. The node will move closer to the center of the cluster and complete the aggregation and mobility behavior finally.

After the above process, the cluster mobility of nodes under the SCM model is finally completed, and all nodes move towards their target nodes at the same time. The flow chart is as follows Fig.

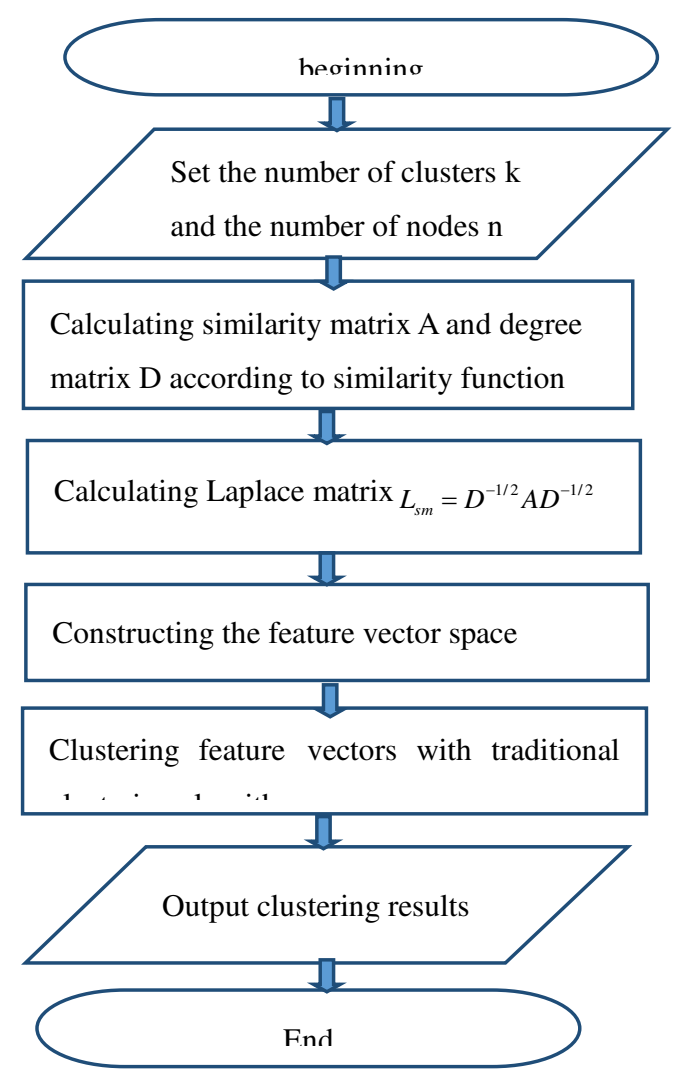

FIG. 1 Flow Chart of Applying Spectral Clustering Algorithm to Cluster Nodes of a Group

\section{SIMULATION EXPERIMENT AND RESULT ANALYSIS}

The simulation software used in this paper is an open source software prepared by the university of Helsinki, Finland, opportunity network simulation simulator[16] (Opportunistic Network Environment Simulator, referred to as "ONE").In order to facilitate performance comparison, the following performance indicators are used:
Delivery Delay, Data packet Delivery ratio, Average hop count, and Overhead ratio of routing protocol.

In this paper, the default Netherlands Helsinki city map is used as the simulation scene of ONE simulator of the group mobility model of SCM spectral clustering algorithm. The simulation parameters in table 1 are the specific settings of SCM spectral clustering model in the scene. For comparison, the configuration of KGM clustering model [17] and SPMB model are the same. In addition, note that the SPMB model is an entity moving model, and there is no 
Table 1 Parameter Setting of Simulation Environment

\begin{tabular}{|c|c|c|}
\hline \multicolumn{2}{|c|}{ parameter } & numerical value \\
\hline \multicolumn{2}{|c|}{ area coverage $\left(\mathrm{m}^{\wedge} 2\right)$} & $4500 \times 3400$ \\
\hline \multicolumn{2}{|c|}{ routing protocol } & Epidemic \\
\hline \multicolumn{2}{|c|}{ simulation time(s) } & 5000 \\
\hline \multicolumn{2}{|c|}{ Maximumcommunication range $(\mathrm{m})$} & 10 \\
\hline \multicolumn{2}{|c|}{ number of nodes(single) } & 200 \\
\hline \multicolumn{2}{|c|}{ cache size $(\mathrm{M})$} & 5 \\
\hline \multicolumn{2}{|c|}{ mobility speed(m/s) } & 6.5 \\
\hline \multicolumn{2}{|c|}{ group number(single) } & 6 \\
\hline \multicolumn{2}{|c|}{ transmission mode } & Simple Broadcast \\
\hline \multicolumn{3}{|c|}{ Table 2 Default Parameter Settings } \\
\hline default parameter & value & description \\
\hline $\mathrm{k}$ & 5 & clustering number \\
\hline $\mathrm{T} 1(\mathrm{~s})$ & 1600 & aggregation start time \\
\hline $\mathrm{T} 2(\mathrm{~s})$ & 4000 & aggregation end time \\
\hline
\end{tabular}

The simulation starts at time 0,200 nodes are randomly distributed on the map, and entity SPMB moves randomly according to their respective Settings. At this time, they have no influence on each other, as shown in figure 2:

After the set clustering time is reached,that is, 1600 seconds later, according to the results calculated by SCM spectral clustering algorithm, all nodes in the network start to move closer to their aggregation center nodes and follow the center point. The aggregation center node itself still moves randomly according to SPMB model. As shown in figure 3, you can clearly see that the nodes on the map have been clustered into five categories.

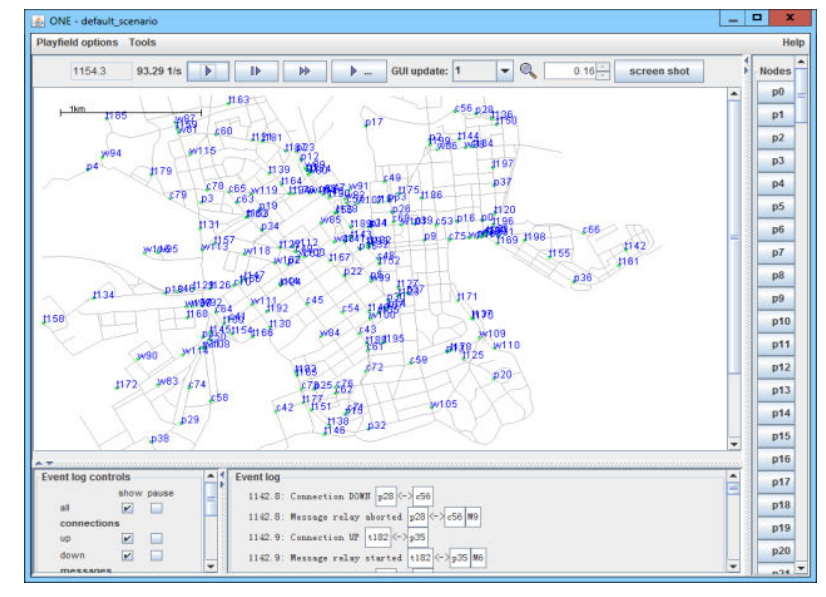

FIG. 2 Random Mobility of Simulation Node

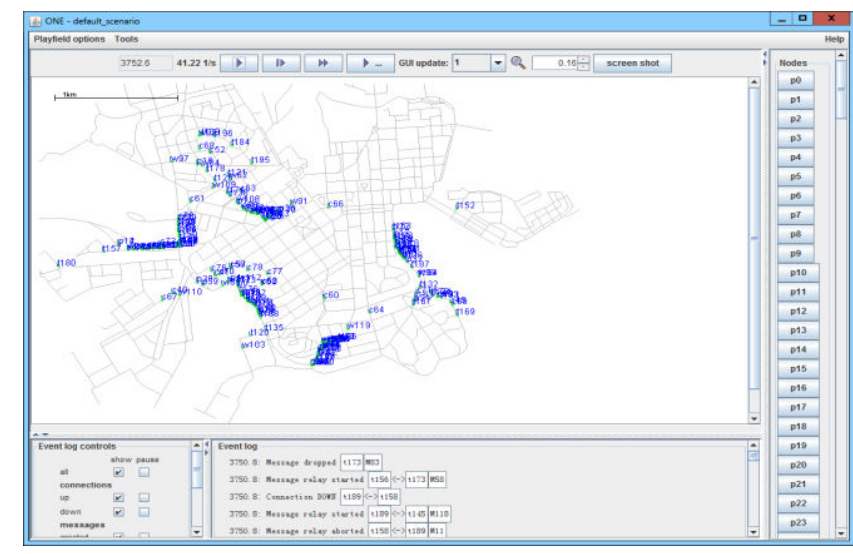

FIG. 3 Group Mobility of Simulation Node After 4000 seconds of simulation, the aggregation process stops, and all nodes start to disperse and continue to move randomly and independently until the simulation ends.

In this paper, the impact of SCM spectral clustering algorithm on the network during node group mobility is verified from two aspects through the simulation of one simulator. The performance of the network is compared according to the different settings of speed.

In experiment 1 we select the formula 1 to calculate the similarity matrix in the SCM algorithm flow and set the value of $\sigma$ manually, so as to verify the influence on the clustering effect of $\sigma$. Traditional SCM clustering algorithm uses Euclidean distance to establish Gaussian 
kernel function to calculate the similarity matrix when constructing the similarity matrix. And $\sigma$ is the scale parameter, which has an important influence on the construction of the similarity matrix and the subsequent clustering results. Here, a number of different $\sigma$ are selected for verification.

Figure 4 shows the relationship between node speed and message transmission rate under different parameters $\sigma$. It can be seen from the figure that the curve of message transmission rate fluctuates greatly with different values of $\sigma$. The result shows that the clustering situation of node group is different with the selection of values of $\sigma$, and the clustering effect is obviously different.

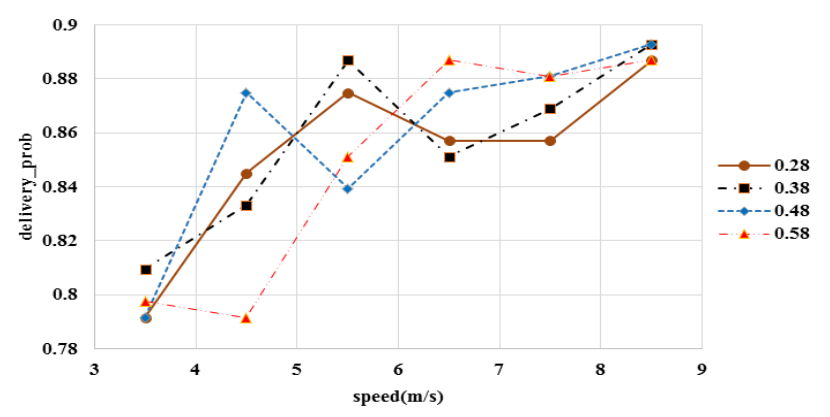

FIG. 4 Relation Between Node Speed and Message Transmission Rate Under Different Parameters $\sigma$

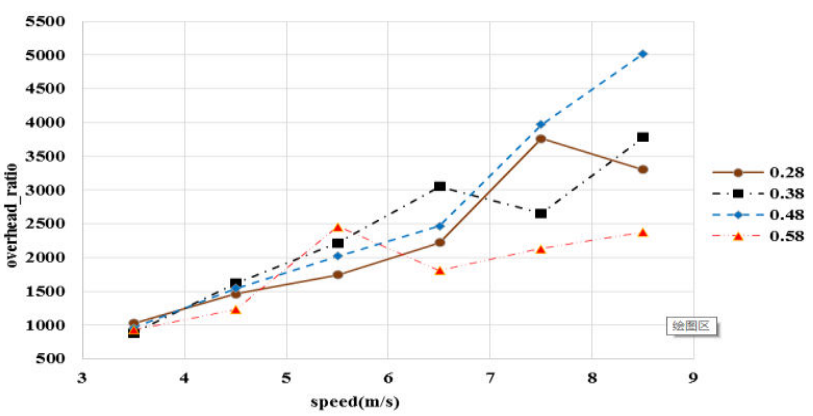

FIG. 5 Relation Between Node speed and Routing Protocol Overhead Under Different Parameters $\sigma$

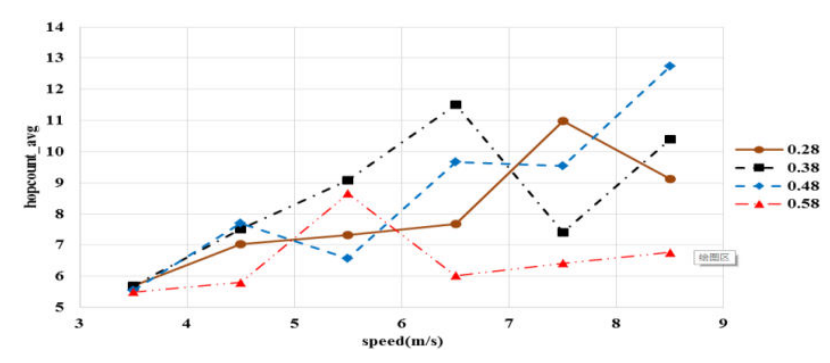

FIG. 6 Relation Between Node Speed and Average Hop Number Under Different Parameters $\sigma$
FIG. 5 and FIG. 6 show the relationship between node speed and routing protocol overhead and average hops under different parameters $\sigma$. That $\sigma$ has an important impact on clustering effect can be verified again. As values of $\sigma$ differs, you can see that the routing protocol overhead and the average hops are quite different from each other. In particular, you can notice that when $\sigma=0.38$ or $\sigma=0.48$, the routing protocol overhead and the average hops increase a bit more than the other two values at the same rate of increase. The routing protocol considered for message transmission between nodes is Epidemic flooding. Therefore, it can be inferred that the reason for the increase is that the nodes gather together in large numbers and move at high speed. It also shows that the SCM clustering effect under these two settings is better than the other two.

In experiment 2, formula 3 was selected to calculate the similarity matrix in the SCM algorithm flow, and the corresponding $\sigma$ value was calculated according to the local node situation. The group mobility model of the adaptive SCM clustering algorithm is obtained. Simulation experiment of this model is compared with KGM model and SPMB model at different speeds. The network performance results are as follows:

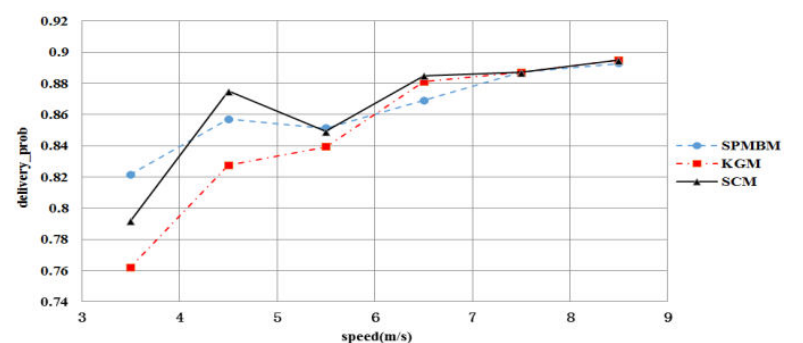

FIG. 7 Relation Between Node Speed of Each

Moving Model and Message Transmission Rate

Figure 7 shows the relation between the speed of each moving model node and the message transmission rate. In the figure, it can be seen that the success rate of message transmission under the SPMB model is relatively stable. Because the nodes in this scenario are independent entities that move randomly, the topology of the whole network is relatively stable. However, compared with SPMB model, the group mobility model based on SCM clustering 
algorithm and KGM clustering algorithm shows a relatively big change in message transfer rate. This is because the nodes have a visible cluster mobility driven by the moving model of the clustering algorithm, resulting in the turbulence in the entire network topology. The message transfer rate of the SCM group mobility model changes faster than that of the KGM model, reflecting the faster clustering speed of the nodes, especially in low speed mobility.

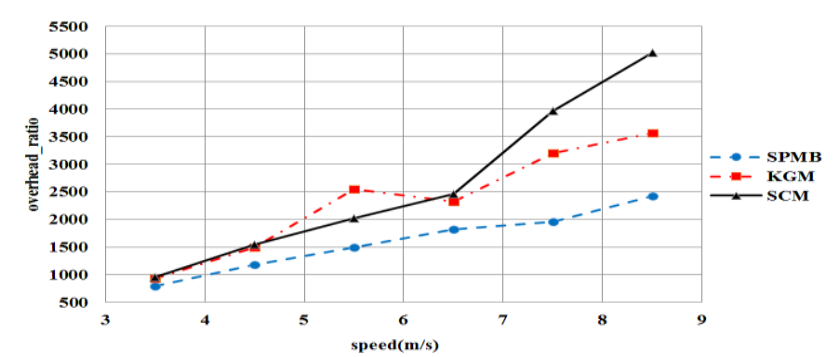

FIG. 8 Relation Between theSpeed of Each Moving

Model Node and the Overhead of Routing Protocol

Figure 8 shows the relationship between the speed of each moving model node and the overhead of routing protocol. In the case of Epidemic routing protocol, as the moving speed of the nodes increases, the overhead of routing protocol is basically on the rise. This indicates that with the increase of node speed, more chances of node meeting are available and forwarding is faster. However, compared with SPMB model, the group mobility model based on SCM clustering algorithm and KGM clustering algorithm shows a relatively large change in message transfer rate. This is because driven by the moving model of the corresponding clustering algorithm, the nodes move in a very obvious clustering group, resulting in the turbulent changes of the entire network topology. Especially in low speed mobility, the message transfer rate of the SCM group mobility model changes faster than that of the KGM model, reflecting the faster clustering speed of the nodes.

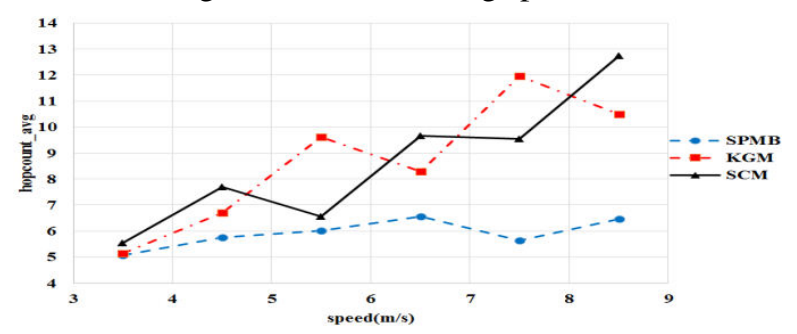

FIG. 9 Relation Between the Speed of Each Moving Model Node and the Average Number of Hops
FIG. 9 represents the relationship between the velocity of each moving model node and the average number of hops. In the SPMB model, the network topology is relatively stable, the node distribution is relatively uniform, and the average number of hops is relatively stable. The SCM model and KGM model have frequent fluctuations in average hops due to the large aggregation of nodes, and the average hops are much higher than those of SPMB model at the same speed.

From the above experiments, it can be seen that the group mobile model based on SCM clustering algorithm not only has flexible property setting, but also better comprehensive performance than the SPMB model and better clustering effect compared with the KGM clustering algorithm model, so it can be studied and applied in many application fields.

\section{CONCLUSION}

Based on the discussion and analysis of traditional group mobility model, this paper presents a group mobility model (SCM) of opportunity network based on spectral clustering algorithm, and improves the node group mobility model. Based on the behavior characteristics of node groups, this paper adopts the method of combining Spectral Clustering algorithm with group mobility model to control the cooperative mobility of a large number of nodes. The algorithm extracts the location information of mobile nodes on the map, uses an adaptive Gaussian kernel function to establish the similarity matrix of moving nodes and applies the Spectral Clustering algorithm for matrix data dimension reduction and cluster analysis. Simulation shows that the SCM model proposed in this paper can describe the node aggregation behavior and group mobility behavior truly and effectively, which shows that the model is suitable for application in some special environments. In addition, the influence of matrix parameter selection on clustering effect under spectral clustering algorithm was verified by experimental simulation, and the performance of Epidemic routing protocol in SCM model was different from that of other models, and the inseparable relationship and importance between mobile model and network routing performance were verified. 


\section{REFERENCES}

[1] Preet Singh T, Singh R K, Vats J. Routing Protocols in Ad Hoc Networks: A Review[J]. International Journal of Computer Applications, 2011, 25(4):30-35.

[2] Kumar M M. A SURVEY ON SENSOR NETWORKS[J]. International Journal of Computer Applications, 2013, 3(2):43-49.

[3] Huang C M, Lan K C, Tsai C Z . A Survey of Opportunistic Networks[C]// 22nd International Conference on Advanced Information Networking and Applications, AINA 2008, Workshops Proceedings, GinoWan, Okinawa, Japan, March 25-28, 2008. IEEE Computer Society, 2008.

[4] MisraS ,Saha B K , Pal S . Opportunistic Mobile Networks: Advances and Applications[M]. Springer Publishing Company, Incorporated, 2016.

[5] Syed Faraz Hasan. Opportunistic Networking[M]. Springer International Publishing, 2014.

[6] Conti M, Kumar M. Opportunities in Opportunistic Computing[J]. Computer, 2010, 43(1):42-50.

[7] Camp T, Boleng J, Davies V. A survey of mobility models for ad hoc network research[J]. Wireless Communications \& Mobile Computing, 2002, 2(5):483-502.

[8] Hong XY, Gerla M, Pei GY, Chiang CC. A group mobility model for ad hoc wireless networks. In: Boukerche A, ed. Proc. of the international Workshop on Modeling and Simulation of Wireless and Mobile Systems. Seattle: ACM Press, 1999:53-60.

[9] Williams S A, Huang D. A group force mobility model[J]. Simulation, 2006.

[10] Ng J M, Zhang Y. Reference region group mobility model for ad hoc networks[C]// Wireless and Optical Communications Networks, 2005. WOCN 2005. Second IFIP International Conference on. IEEE, 2005:290 - 294.

[11] Chang Y C, Liao H C. EMM: an event-driven mobility model for generating mobilitys of large numbers of mobile nodes[J]. Simulation Modelling Practice \& Theory, 2005, 13(4):335-355.
[12] Ng A Y, Jordan M I, Weiss Y. On Spectral Clustering: Analysis and an algorithm[J]. Proceedings of Advances in Neural Information Processing Systems, 2002, 14:849--856

[13] Ding S, Zhang L, Zhang Y. Research on Spectral Clustering algorithms and prospects[C]// International Conference on Computer Engineering and Technology. IEEE, 2010:V6-149-V6-153.

[14] Bettstetter C , Resta G , Santi P . The Node Distribution of the Random Waypoint Mobility Model for Wireless Ad Hoc Networks[J]. IEEE Transactions on Mobile Computing, 2003, 2(3):257-269.

[15] Chi, Yun, Song, et al. Evolutionary spectral clustering by incorporating temporal smoothness[C]// ACM SIGKDD International Conference on Knowledge Discovery and Data Mining, San Jose, California, Usa, August. DBLP, 2007:153-162.

[16] Ari Keränen, Jörg Ott, TeemuKärkkäinen. The ONE simulator for DTN protocol evaluation[C]// Proceedings of the 2nd International Conference on Simulation Tools and Techniques for Communications, Networks and Systems, SimuTools 2009, Rome, Italy, March 2-6, 2009. ICST (Institute for Computer Sciences, Social-Informatics and Telecommunications Engineering), 2009.

[17] Li Y , Wu H . A Clustering Method Based on K-Means Algorithm[J]. Applied Mechanics and Materials, 2012, 25(none):1104-1109.

\section{Biographies and Photographs}

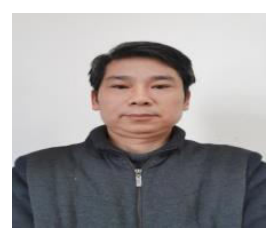

Hong $\mathrm{He}$ received his B.S. degree atWuhan University of Technology in 1995, and M.S. degree at Xiangtang University in 2006.Currently, he works in Hunan Institute of Engineering as an associate professor. His research interesting is data mining, grid computing, cloud computing,distributed resource management. 